CERN EP/2008-004

21 February 2008

\title{
1980, A REVOLUTION IN SILICON DETECTORS, FROM ENERGY SPECTROMETER TO RADIATION IMAGER: SOME TECHNICAL AND HISTORICAL DETAILS
}

\section{ERIK H.M. HEIJNE, CERN PH - DEPARTMENT}

CH1211 Geneva 23 Switzerland

\begin{abstract}
Silicon nuclear particle detectors were introduced just 50 years ago, after single crystal manufacturing was mastered. A major change took place around 1980 when the 'planar' MOS (Metal Oxide Semiconductor) technology developed in microelectronics was systematically applied also in detector construction. With the simultaneous introduction of matched readout chips this eventually would lead to pixelized matrix detectors that function as radiation imaging devices. The critical contributions to this revolution by Josef Kemmer and Paul Burger are described. Performance of the segmented planar technology detectors improved significantly in comparison with the earlier spectrometric diodes. With efficient industrial support the use of silicon detectors in many new applications has become possible and detector systems with a sensitive area of several tens to $>100 \mathrm{~m}^{2}$ have been constructed recently.
\end{abstract}

PACS: $29.40 ; 07.90 ; 87.53 ; 01.50 ; 14.60$

Keywords: Silicon detector; Radiation imaging; Low noise; History of semiconductors

Submitted to Nucl. Instr. Meth. A for Proceedings of 9th IWoRID, Erlangen, 22-26 July 2007 


\section{1980: silicon detectors before and after}

Silicon detectors originated about 1957, and in a sense they became adult around 1980. At that time the technological innovations in sensor manufacturing and readout circuits started a period of renewed interest and large-scale applications, particularly in space instruments and in particle physics experiments. These developments later resulted in real 2-D imaging capabilities for particle and X-ray radiation. This article describes some technical and historical details of the silicon detector history and the revolution of 1980. It is mostly based on publications, augmented with recollections and a personal view on events in which the author participated. Special attention is given to not-so-widelyknown contributions by Dr Paul Burger, at the occasion of his retirement in June 2007. He is the Guest of Honor of this 9th International Workshop on Radiation Imaging Detectors in Erlangen. However, from the outset, the author wishes to stress that developments such as described here are the result of efforts by many people. They study, they meet, they get ideas and make new devices, put new technologies to work. Intentionally or serendipitously they reinforce each others contributions and suddenly a revolution in the field takes place. The introduction of silicon planar technology ${ }^{1}$ for detectors was such a revolution which could have been predicted, yet took many by surprise. Several contributing scientists will be mentioned. In a few pages it is impossible to do justice to all who played a role, and the author welcomes further comments because this article is certainly not the 'ultimate history' on the subject ${ }^{2}$.

As soon as single-crystal silicon became available in the early fifties, it has been used for nuclear particle detection and spectroscopy. In contrast with devices for electronic functions (diodes, transistors) where only a thin surface layer, few $\mu \mathrm{m}$, of the silicon is used, nuclear radiation detectors need thick sensitive volumes with a low concentration of free charge carriers, so that the radiationgenerated charge signal can be detected above the background. The earliest structure was a photoconductor with high resistivity obtained by gold-doping [1]. It followed the priniple of the $\mathrm{AgCl}$ crystal counter from van Heerden in 1943 [2]. Already for his Ge counter McKay in 1949 [3] was aware that a rectifying junction (a point-contact 'barrier') is needed to achieve a low dark current and a depleted volume in a semiconductor, even if it was still tiny in his case. In $\mathrm{Si}$, at first diffused np junction devices were used [4], sometimes mesa-type but also with oxidized edges [5]. Schottky surface barrier diodes were also employed. A detailed record of the early discussions on problems and relative merits of different structures can be found in the USA National Academy of Sciences publications from 1961[6] and 1969[7]. By 1965 the surface barrier structure became widely preferred, because of low noise, low reverse current and the thin layer contact structure, causing only a

\footnotetext{
1 The word 'planar' may be confusing because it has been used also to indicate detectors with parallel contact surfaces, in contrast, for example, with a coaxial germanium detector, which has a central hole contact and an outer contact as a continuous cilinder or segmented facets. Silicon detectors practically always have been 'planar' in this sense, but their manufacturing by 'planar' technology refers to the use of the specific processing steps developed for silicon integrated circuits.

2 Sherwood Parker (sher@slac.stanford.edu) also has a long-time interest in the history of the silicon microstrip detector and the associated integrated circuits for the readout. He and the author collect historical and technical information and even sample artefacts.
} 
small energy deficit from the entrance window. For the best energy resolution in X-rays and $\gamma$-rays the large volume Ge detectors remain unsurpassed, but for many X-ray applications the more affordable lithium-drifted, thick silicon detector can be sufficient, usually with liquid nitrogen cooling.

New technologies such as ion-implantation have been tried as early as 1962 by Alväger and Hansen in Argonne [8], in 1967 by Meyer and Haushahn in Karlsruhe [9], James Mayer at Caltech / Hughes Aircraft [10] and Zandveld at Philips [11]. These devices apparently did not show convincing advantages compared with the commercially available Si surface barrier detectors and their promise has been fulfilled only much later. While numerous laboratory teams continued to fabricate their own detectors, several companies in the USA and in Europe could supply high quality silicon nuclear detectors for spectroscopy or special applications. A non-exhaustive list based on my perspective in 1975 is shown in table 1 in section 3.

During the seventies the silicon integrated circuit started to drive a faster than exponentially growing industry. Semiconductor research institutes and manufacturers made enormous progress in silicon processing technology. With continuously improved Si oxidation one achieved reproducibility and sub-micrometer precision in the definition of diodes and transistors. The related key technologies are photomask lithography, junction formation by ion-implantation and contacting with etched metal lines. Several people independently thought about the impact that this might have on the nuclear detector fabrication, which for the moment seemed to have become a mature field, but employing early, by then rather conservative technology. During the 1979 IEEE Nuclear Science Symposium in San Francisco I had a discussion on this with Paul Burger (over a dinner in the Japanese restaurant Yamato), and he mentioned that his company Enertec planned to fabricate detectors based on such planar technology in the new Lingolsheim facility. Since some time he collaborated with Josef Kemmer, working at the Technische Universität München in Garching, on an oxidation process that would allow diodes to be made by ion implantation. In early 1980 Kemmer published first results from this process [12]. At $100 \mathrm{~V}$ reverse bias, at total depletion, he could achieve dark current close to one $\mathrm{nA}$ per $\mathrm{cm}^{2}$ per $100 \mu \mathrm{m}$ thickness of $\mathrm{Si}$, which is indicative of a minority carrier generation lifetime of $>15 \mathrm{~ms}$, longer than that of the starting Si wafer. In the corresponding patent application [13] a more detailed description was given of an oxide edge profiling that improves the breakdown characteristics. Discussion on technical aspects of this work follows in the next section. The visionary contribution by Paul Burger in 1980 was the transformation of the laboratory-scale process of Kemmer into a comprehensive, commercially accessible chain from detector design, mask supply and industrial manufacturing. This enhanced the impact of the work by Josef Kemmer, and the implementation in the commercial environment of Enertec-Schlumberger provided access to the new planar detectors for a variety of new customers. It is a testimony to the success of this initiative that soon afterwards several other enterprising and innovative people entered in this activity, either within an existing company, e.g. Kouei Yamamoto from Hamamatsu, or starting a completely new company, in particular Colin Wilburn who in 1983 founded Micron Semiconductor Ltd. The creation of several commercial suppliers provided a stimulus for quality and a welcome guarantee for the potential users. Also, contrary to previous widespread practice in nuclear science, this situation made it unnecessary to install significant 
production lines in the research facilities of the users themselves. It turned out that the time was ripe for a new generation of radiation imaging devices that could provide new tools for new science. The development of relatively affordable signal processing circuits was certainly an important enabling condition, which had been pioneered since 1970 in particle physics experiments for MultiWire Proportional Chambers (MWPC).

The main result of the Yamato meeting mentioned above, was that Pierre Jarron and I set out to organize in collaboration with Paul Burger the design and production of a prototype 100-strip, $200 \mu \mathrm{m}$ pitch rectangular silicon diode array detector, which we dubbed 'microstrip detector', at first still using the well-mastered surface barrier technology [14]. But already by the end of 1980 these surface barrier rectifying elements were replaced by diode elements made with the planar approach [15]. Pierre designed a miniature analog readout system with a fast amplifier, based on a recent Philips bipolar microwave transistor. This microstrip detector system was first shown to work in a test beam in the CERN South Hall in April 1980, with the support of François Piuz, and then used for two 8hour shifts in May behind the target of the NA11 experiment in the North Hall H6 beam, in collaboration with Bernard Hyams and his postdocs Jos Vermeulen and Andrew Wylie. Using the combination of data from wirechambers in the existing spectrometer and a single new microstrip detector we could demonstrate immediately the reconstruction of an interaction vertex[14] with improved accuracy. The possibility to distinguish a secondary vertex naturally triggered the interest of the NA11 collaboration. Several references to earlier implementations of such segmented silicon detectors have been given in [14]. It should be emphasized that the double-sided microstrip detector, the Si 'checker board,' had been patented by Philips collaborators in Amsterdam as early as 1966 [16,17]. René van Dantzig in 1977 had been instrumental in showing Pierre and myself at IKO in Amsterdam the details of the approach [18]. Earlier in 1980 an effort had already been underway independently in Italy, where the INFN-Pisa group developed with Filippo Nava (from the semiconductor detector group in Modena) a Si surface barrier multi-electrode detector with 19 strips at $600 \mu \mathrm{m}$ pitch. They measured signals with this device at CERN in March 1980 [19] but did not yet demonstrate vertex reconstruction as we did two months later [14]. Some of these early results with $\mathrm{Si}$ detectors for elementary particles were discussed at the first 'Pisa' instrumentation conference in September 1980, in Tirennia [20]. A year later, there were already several conferences and symposia dedicated to silicon devices for charm physics, such as the Fermilab Workshop organized by Tom Ferbel [21] and the Europhysics Conference in Erice, organized by Antonino Zichichi [22].

In August 1980 the NA11 collaborators in Munich, Robert Klanner and Gerhard Lutz in particular, initiated independently from Enertec a collaboration with Josef Kemmer and by 1982 they had constructed a silicon telescope with six detectors [23]. Soon afterwards they could show the first pion interaction with a secondary D decay vertex reconstructed with the silicon microstrip detector telescope [24]. This certainly became the most convincing piece of evidence for the particle physics community. In November 1983 the Munich group resuscitated the European Semiconductor Detector Symposium [25], where they brought together all people then working in the field. 
Actually ahead of the developments in Munich, Paul Burger with Josef Kemmer and I had been designing and producing a series of planar technology, ion-implanted microstrip detectors as the successors to the $200 \mu \mathrm{m}$ and $50 \mu \mathrm{m}$ pitch surface barrier microstrip devices. At the Europhysics Conference in Erice in November 1981, Paul Burger introduced the commercial availability of this planar technology based on the work by Josef Kemmer [26]. The first ion-implanted microstrip detectors from Enertec were tested at CERN $[15,26]$ and were subsequently employed in the WA75 experiment by Paul Musset and François Piuz, and in the Omega spectrometer by the WA82 collaboration with Diambrini-Palazzi for the location of vertices in an emulsion [27].

In the USA there had been discussions at Brookhaven National Laboratory in view of the needs for such precision detectors at the Isabelle hadron collider [28] and also at BNL a study on segmented Si detectors was published by Kanofsky already in 1977 [29]. The Fermilab Workshop organized by Tom Ferbel in October 1981 [21] was instrumental in directing efforts towards development and physics applications of silicon detectors. BNL made the first practical steps in 1982 [30]. Unlike most European predecessors, BNL as well as Lawrence Berkeley Laboratory during the 80s built up a Si detector manufacturing facility for technology study and prototyping.

The conjunction of the planar technology, the re-introduction of the microstrip sensor and the miniaturization of the readout electronics created the fertile ground for the Si detector revolution and it was recognized that this would be just the instrument for the new needs in particle physics. The main achievement is the transformation from a single-particle spectrometric device into a large-area multiparticle imager with micrometric precision for simultaneous hits and nanosecond time resolution. The lithographic method allows a variety of small size, high precision elements of the silicon sensor matrix. It became immediately clear that using segmentation, a much lower noise can be achieved and that charge division between small segments can be exploited to improve localization of the ionizing particles. The capability to provide image reconstruction of elementary particle interactions with precision of $\sim 10 \mu \mathrm{m}$ led to widespread use of silicon detector arrays. These allowed the study of the recently discovered short-lived elementary particles with charm and beauty quarks. The apparatus used previously, such as bubble chambers or Multi-Wire Proportional Chambers did not have sufficient precision or speed.

The new age of silicon detectors has seen a large increase in the size of a typical detector system, from a few $\mathrm{cm}^{2}$ in a usual nuclear experiment, to over hundred $\mathrm{m}^{2}$ in the large collider experiments of this decade. Also space-borne experiments have adopted these large Si detectors, such as for example AMS with $8 \mathrm{~m}^{2}$ [31] and GLAST with $80 \mathrm{~m}^{2}$ [32]. With these new applications and the more efficient manufacturing process the business volume in Si particle detectors has grown from a few M\$ per year around 1975 to some tens of $\mathrm{M} \$$ in 2005 . At the same time the unit cost per Si detector has been reduced by a factor 5-10, and the cost per sensor channel, strip or pixel by several orders of magnitude. The even much larger cost reduction per function observed for integrated circuits is achieved by building the same or more functions on a much smaller Si area, but this is not possible for particle detectors. On the contrary, new applications tend to need much larger Si area, and therefore a cost reduction can only come from a more efficient production, not from the use of less silicon. In 
parallel, traditional applications of Si detectors have also taken profit from the introduction of the planar technology, mostly by improved noise performance, reliability and ruggedness. Planar ionimplanted devices have a surface that can be cleaned and they also can be recycled at elevated temperature after radiation damage, if the packaging is adapted for this.

Precision lithography on both sides of the high resistivity silicon wafer allows the application of a lateral electrical drift field on top of the depleting voltage. Based on this principle, the silicon drift detector has been introduced by Emilio Gatti and Pavel Rehak [33]. This concept of continuous (not clocked) signal charge transport over a longer distance via a field valley deep in the bulk is similar to the Resistive Gate Device [34] which also used charge transport via a field gradient close to the surface for visible light imaging. The clocked Charge Coupled Device structure has become preferred for visible imaging, but for energetic radiation the drift detector is more used than high resistivity, deep depletion CCD. Both structures offer a small capacitance output node and therefore achieve excellent signal/noise ratio as shown in section 2.1.

\section{2 . The planar silicon detector ${ }^{3}$ technology}

The essential feature of planar silicon technology is the application of successive processing steps on the surface of a silicon wafer [35]. The silicon oxidation with subsequent lithograpy of a pattern is the most important process [36]. As a consequence the quality of the $\mathrm{SiO}_{2}$ (amorphous 'quartz') layer and the $\mathrm{Si}-\mathrm{SiO}_{2}$ interface are critical and $\mathrm{SiO}_{2}$ continues to be intensively studied. Early transistors, in contrast, were made by methods such as alloying of pieces with different doping levels, and also operations of ultrasonic cutting of mesa-type devices or edge protections with epoxy glues were not 'planar'. The planar technology allowed to replace discrete devices by Si chips with many devices wired together as an integrated circuit [37]. In a similar way, the use of planar technology in detector manufacturing allows to make a contiguous matrix of small diodes with micrometer precision. Moreover, by perfecting planar technology processing methods, the performance and reproducibility of devices has improved tremendously and this has created the possibilty of large-area manufacturing and new applications.

\subsection{Detector noise}

The basic quality criterium for silicon diode particle detectors is the noise in the signal charge collection. This depends on the detector geometry, collecting electrical field strength, the purity of the detector volume, i.e. absence of charge trapping centers, and the diode reverse current. The influence of this current is discussed in section 2.2. The effective noise observed also depends on the characteristics of the signal processing circuit, and in particular on the shaping time and the noise parameters of the first transistor, which should be matched to the detector capacitance.

Customer specifications for Si surface barrier detectors usually centered on noise performance in alpha-particle spectroscopy, with the best, smallest devices until 1980 achieving 15 keV FWHM

\footnotetext{
3 See footnote 1 on first page
}

6 
(corresponding to an Equivalent Noise Charge ENC $1760 \mathrm{e}^{-} \mathrm{rms}$ ) for $\sim 5 \mathrm{MeV} \alpha$-particles. The reverse bias dark current of 0.5 to $1 \mu \mathrm{A}$ per $\mathrm{cm}^{2}$ is one of the factors that limit this noise performance, but also detector capacitance, 1/f noise, charge trapping and other effects degrade spectroscopic quality.

The introduction of the planar technology, the Si detector 'revolution,' improved the noise performance in various ways and Kemmer, Burger et al. $[12,15,22]$ reported already $10 \mathrm{keV}$ FWHM for ${ }^{241} \mathrm{Am} \alpha$ particles and $1.55 \mathrm{keV}$ for ${ }^{57} \mathrm{Co}$ and ${ }^{241} \mathrm{Am} \mathrm{X}$-rays. The dark current per $\mathrm{cm}^{3}$ has been reduced (section 2.2), but also the surface noise is better controlled by the oxide edge protection. A fundamental improvement has been achieved by the drift detector design, or by the segmentation of a large surface area in a matrix of small-capacitance, small area 'pixel' diodes. Each of the segmented diodes achieves a noise that in principle is lowered by the factor $\sqrt{ }\left(\mathrm{C}_{\text {segment }} / \mathrm{C}_{\text {matrix }}\right)$ if the total power is kept constant. In the Si drift detector one or several small-capacitance nodes provide the low-noise output for a relatively large sensitive detector area, and with this device it has become possible to detect X-rays of a few $\mathrm{keV}$ with noise less then $40 \mathrm{e}^{-} \mathrm{rms}(0.35 \mathrm{keV} \mathrm{FWHM} \mathrm{in} \mathrm{Si})$ at room temperature [38]. This performance approaches that of thick Li drifted Si X-ray detectors which achieve $\sim 0.2 \mathrm{keV}$ FWHM at cryogenic temperature. Even lower noise is possible if the dark current can be limited further, and $20 \mathrm{e}^{-} \mathrm{rms}$ was obtained by the same authors at $-35^{\circ} \mathrm{C}$, with dark current $0.1 \mathrm{pA} \mathrm{cm}-2$ but then various sources of dielectric noise are the limit rather than the detector current [38]. In 1994 the noise performance of a planar Si detector for protons, deuterons and alpha has been studied by Steinbauer et al [39] and they report measured values between $3.6 \mathrm{keV}$ FWHM $\left({ }^{1} \mathrm{H}\right)$ and $10 \mathrm{keV}$ $\left({ }^{4} \mathrm{He}\right)$, which appear to be close to the ultimate physical limits.

\subsection{Detector operation voltage, diode reverse current, contacts and segmentation}

The reverse bias voltage applied between the rectifying contact and the rear ohmic contact serves to deplete the volume from free charge carriers, and to establish a strong signal charge collection field, which e.g. in a

$\mathrm{p}^{+} \mathrm{n}$ diode sweeps the holes towards the collecting diode contact. The electrical field should be as strong as possible, the diode avalanche breakdown field in Si being $\sim 3 \times 10^{5} \mathrm{~V} / \mathrm{cm}$, but between 15 to $30 \mathrm{kV} / \mathrm{cm}$ $(3 \mathrm{~V} / \mu \mathrm{m})$ the drift velocity saturates $\left(80 \mu \mathrm{m} / \mathrm{ns}\right.$ for $\mathrm{e}^{-}$at $\left.300 \mathrm{~K}\right)$ and no further increase in speed is achieved. However, it is rare that one can actually apply such a bias voltage of $900 \mathrm{~V}$ to a $300 \mu \mathrm{m}$ thick, standard $\mathrm{Si}$ detector, because breakdown or rear-contact minority carrier injection often occur already at a lower voltage due to various imperfections. Also, the packaging of the device has to take into account that the breakdown voltage in air is only $\sim 3.3 \times 10^{4} \mathrm{~V} / \mathrm{cm}$. Special care has always been taken to provide protection at the diode edge, where surface leakage current, breakdown and noise are easily generated. The edge processing and glueing is a well-guarded manufacturing secret for the surface barrier devices. Sharp corners have to be avoided for optimal breakdown characteristics, and also in view of the high cost of the Si material the standard detectors in the beginning were all circular, as cut from the grown single crystal. High-resistivity $\mathrm{Si}$ monocrystalline ingots were purchased by the detector manufacturer, usually one inch diameter, with the slicing, lapping and polishing done in-house. Sometimes even the crystal growing and zone refining were done by the teams themselves. All this has changed after the introduction of the planar technology, for which commercial high resistivity $\mathrm{Si}$ wafers are purchased, of standard diameters $75 \mathrm{~mm}$ up to 
$200 \mathrm{~mm}$, mostly with single-sided polishing, and lapped or polished rear surface. The wafers are then completely oxidized in a furnace and windows are etched for the diffusion or ion-implantation that create the $\mathrm{p}^{+} \mathrm{n}\left(\right.$ or $\left.\mathrm{n}^{+} \mathrm{p}\right)$ diode junctions. With photoresist lithography it has become much easier to design arbitrary shapes for the detecting area, and in particular this resulted in matrix detectors with pads, narrow microstrips or small pixels. In Fig. 1 a schematic overview of a few different edge terminations for such oxide passivated junctions is presented.

a)

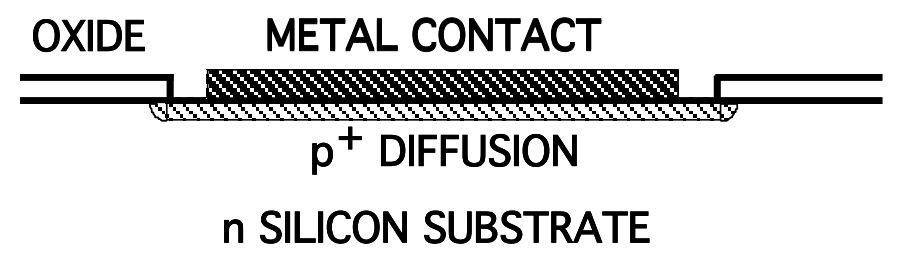

b)

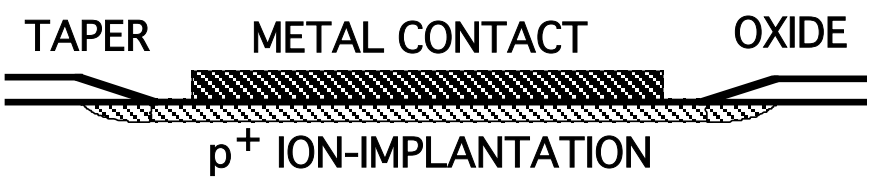

n SILICON SUBSTRATE

c)

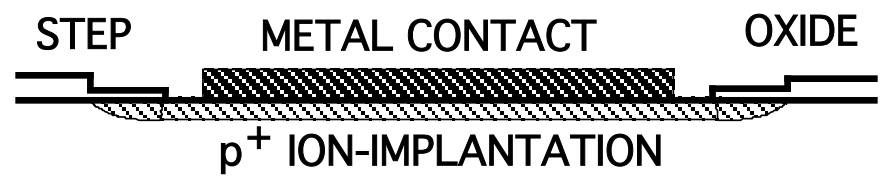

n SILICON SUBSTRATE

d)

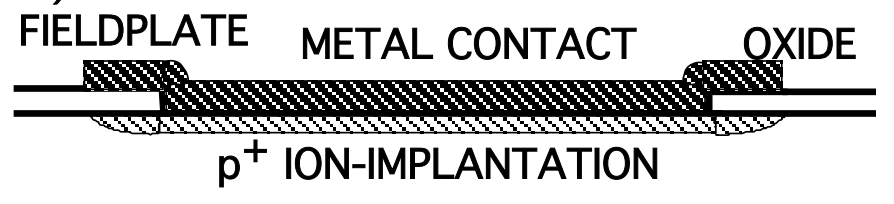

n SILICON SUBSTRATE
Fig. 1 Schematic diagrams of different $\mathrm{Si}$ detector edge architectures. The drawings are not to scale. Often the thickness of the field oxide for detectors is $\sim 300 \mathrm{~nm}$, the depth of a difusion can range from $200 \mathrm{~nm}$ to microns, and a low energy ion-implantation may be as shallow as $50 \mathrm{~nm}$. The aluminium metal contact is often $\sim 400 \mathrm{~nm}$ but may be thinner if low-energy particles must pass through.

a) simple oxide passivation as in [5]

b) tapered oxide creates gradual doping concentration profile in the $\mathrm{Si}$ under the edge after ion implantation [11]

c) oxidestep with similar purpose [13]

d) metal contacts extending onto the field oxide also lower the electrical field gradient at the edge, as studied by Conti and Conti [40]. Note that fieldplate does not refer to an applied electrical field but to the position of the plate on the field oxide 4

Numerous efforts have been made to improve the breakdown characteristics of the diodes, and as mentioned already, the 1980 patent by Kemmer [13] aimed at high breakdown by etching a step in the field oxide layer that delimits the rectifying area. This step is etched before the ion-implantation process and causes a gradient in the doping density around the junction edge, as schematically illustrated in Fig.1c. This oxide step profile patented by Kemmer can be considered a simplified version of the earlier patent by Zandveld [11]. Zandveld also intended to lower the field strength at the diode edge by a gradual doping profile of the ion implantation, but using a beveled or tapered oxide edge, illustrated in Fig.1b. He could apply voltages in excess of $1000 \mathrm{~V}$ with a combination of the tapered edge and a guard ring. Much more detailed studies of the breakdown voltages and edge profiles have been done for $\mathrm{Si}$ power devices as described by Baliga [41] and using more

\footnotetext{
${ }^{4}$ The word field in 'field plate' refers to the 'field oxide' and has the meaning as in 'field of view' or agricultural field, in which some useful features such as transistors will be placed. The term here does not refer to 'electric field'. Confusion may arise because sometimes but not necessarily an electric potential may be applied to such a 'field plate'. 
homogeneous high-resistivity Si (by Neutron Transmutation Doping NTD) one can achieve several $\mathrm{kV}$ operating voltage, but the penalty is the need for a structure of guard rings. In Si detector arrays such guard rings present undesirable dead area, and recently much study is invested in edge-less detector designs. Chris Kenney, Sherwood Parker et al. proposed in 2005 an edge-less ion-etched structure [42]. They have used this ion-etching technology also to make a pillar matrix in the silicon, called the 3-D detector, that provide s a strong lateral collecting field even at low bias voltage [43].

Kemmer was the first to report reverse bias leakage currents below $5 \mathrm{nA} \mathrm{cm}-2$ at $100 \mathrm{~V}[12,15]$. For some time it was not clear if this low dark current could be attributed to the edge profiling, or if it had been achieved by additional processing details. Some years earlier Keil and Lindner [44] also used oxide edge passivated detectors, but they obtained at best a reverse current $\sim 60 \mathrm{nA} \mathrm{cm}-2$. Similarly, Zandveld provides many more details [11], yet he quoted a reverse current of some $\mu \mathrm{A}$ per $\mathrm{cm}^{2}$ at $100 \mathrm{~V}$ [11, Fig11]. It seems that improvement in processing equipment and ultra-clean room environment is the main factor towards defect-free oxidation with low interface charge. Additional reduction of generation-recombination impurity centers by gettering and use of $\mathrm{HCl}$ or TCE (TriChloroEthane) in the oxidation ambient also play an important role. Use of $\mathrm{HCl}$ in the oxidation process has been mentioned both by Zandveld and Kemmer. Following the formula for generation current $\mathrm{I}_{\mathrm{G}}$ one can estimate the minority carrier lifetime $\tau$

$\mathrm{I}_{\mathrm{G}}=\left(\mathrm{n}_{\mathrm{i}} / 2 \tau\right) \mathrm{qwA}$

with $\mathrm{n}_{\mathrm{i}}=1.5 \times 10^{10} \mathrm{~cm}^{-3}$ and $\mathrm{q}=1.6 \times 10^{-19} \mathrm{C}$. The sensitive volume is thickness $\mathrm{w}$ times area A. Kemmer [11,15] derives a carrier lifetime of the order of $15 \mathrm{~ms}$, which is much higher than that determined for the starting Si wafer, and indicates a very effective impurity and defect gettering. The $\mathrm{I}_{\mathrm{G}}$ is only one of the components of the leakage current, besides surface generation, leakage and diffusion current from undepleted parts. Therefore the estimated $\tau$ is a lower limit.

The fluctuations in the reverse current are proportional to its magnitude $\mathrm{I}_{\mathrm{D}}$ and introduce a parallel or current component in the equivalent noise charge $\mathrm{ENC}_{\mathrm{p}, \mathrm{i}}$ which can be reduced by a short amplifier shaping time $\tau_{\mathrm{s}}$ as long as this is compatible with the signal collection time

$\mathrm{ENC}_{\mathrm{p}, \mathrm{i}}=\sqrt{ }\left[(2 / \mathrm{q}) \mathrm{K} \mathrm{I}_{\mathrm{D}} \tau_{\mathrm{s}}\right]$

$\mathrm{K}$ is a constant between 0.3 and 1 , depending on the shaping filter. This noise component becomes less important with the low currents achieved in the new planar processing, and can be further reduced by segmentation of the sensor. A lower limit for the equivalent noise charge ENC depends on the dark current and $100 \mathrm{e}^{-} \mathrm{rms}$ can be obtained if this is $<1 \mathrm{nA}$. Lower values can be reached by further optimization of the sensor (with segmentation or drift) and of the readout electronics [45].

The role of the 'rear' ohmic contact for the mastering of leakage current and breakdown is considerable, and the introduction of planar technology has provided a much better control of this aspect of the manufacturing. The implantation of a non-equilibrium dose of low energy As ions allows a very thin, strongly $\mathrm{n}^{+}$type sheet contact, probably degenerate, where the concentration and the lifetime of the minority hole carriers are small. Then there will be no injection and diffusion current. 
The study of Rawlings et al. at Harwell in 1986 [46] concluded that this high-low same-type junction was the key to the low leakage current of the Kemmer process, and that of later commercial suppliers. Nowadays, this performance is taken for granted, although it may be compromised by inadequate dicing, scratching, etc.

Segmentation of the detector area has been emphasized as a means to obtain low amplifier input capacitance, small leakage current and consequently low noise. More important, the production of linear microstrip detectors and 2D matrix pixel detectors has led to many new applications where the measurement of the position of the incoming radiation is essential. Before the planar approach with multiple segments the preferred method for position measurement was resistive charge division, as reviewed by Laegsgaard [47]. In a typical device with a few $\mathrm{cm}$ length, one could achieve a precision of the order $1 \%$, corresponding to a few tenths of $\mathrm{mm}$, depending on the signal/noise ratio. In a microstrip detector, with dimension $>8 \mathrm{~cm}$ for a single device, one can now readily obtain a precision $<20 \mu \mathrm{m}$, even for multiple, simultaneously incident particles.

\section{3 . Industry and the silicon detector $R \& D$}

Commercial manufacturing has been crucial for the development of Si detector . Large scale silicon arrays such as needed in space instruments and in particle physics experiments can not be built without dedicated manufacturing facilities and experienced staff. On the other hand, the research on semiconductor devices has been largely done in universities and public and industrial research institutes. All the time there has been a transfer towards commercial suppliers, and these also have contributed in essential ways to the continued development of device technology. The manufacturability has been an important point, as well as yield. Over the years there have been numerous suppliers, which were often founded by scientists who had their origin in a nearby laboratory. ORTEC was founded as one of the first commercial suppliers, by scientists who had worked at the Oak Ridge Labs. A tentative and rather subjective list of companies that existed around 1975 is shown in the upper half of Table 1 . The list is based on a market survey that CERN made in view of the supply of $\sim 300$ silicon detectors for the SPS neutrino beam monitoring system. It can be noticed that most suppliers then produced surface barrier detectors. Some companies were mostly producing for their internal needs, such as Siemens for their 'air ball' nuclear reactor neutron monitoring systems, and Philips for their electron microscopes. The Philips 'Checker Board' detector [17] was practically the only segmented Si detector for sale, but it was difficult to build a readout system for such a device.

The second part of the table shows some of the commercial suppliers which existed around 2000, just when the discussions started for the manufacturing of the silicon detctors for the Large Hadron Collider at CERN. Since then several additional suppliers have come into operation as well. Now nearly all manufacturing is based on the planar technology. Already before, it was mentioned that the essential contribution in 1980 by Paul Burger has been to transform the laboratory-scale planar 
process by Josef Kemmer for the first time into a complete industrial detector manufacturing process. Now this technological approach has become commonplace, for the benefit of the community.

Table 1 Some commercial suppliers (alphabetical) of silicon radiation detectors $\sim 1975$ and $\sim 2000$

\begin{tabular}{|c|c|c|c|}
\hline $\begin{array}{c}\text { Company } \\
1975\end{array}$ & City & Main Si Products & Contact Person \\
\hline LASCO & Strasbourg & $\begin{array}{l}\text { Si surface barrier, } \\
\text { readout modules }\end{array}$ & R. Henck, P. Burger \\
\hline NUTRONICS & London & Si surface barrier & \\
\hline ORTEC & Oak Ridge & $\begin{array}{c}\mathrm{Si} \\
\begin{array}{c}\text { surface barrier, } \\
\text { readout modules }\end{array}\end{array}$ & $\begin{array}{l}\text { F.J. Walter, } \\
\text { M.Trammel, }\end{array}$ \\
\hline PHILIPS & Eindhoven & $\mathrm{Si} \begin{array}{c}\text { surface barrier, } \mathrm{Si}(\mathrm{Li}) \text {, } \\
\text { incompany use mostly }\end{array}$ & $\begin{array}{l}\text { A. Hoeberechts, } \\
\text { R.Beeftink }\end{array}$ \\
\hline QUANTRAD & Los Angeles & Si diffused & F. Ziemba \\
\hline SIEMENS & Erlangen & $\begin{array}{l}\text { Si surface barrier } \\
\text { incompany use only }\end{array}$ & P. Glasow \\
\hline SIMTEC & Chalk River & $\begin{array}{l}\text { Si diffused, } \\
\text { readout modules }\end{array}$ & M. Martini \\
\hline 2000 & & & \\
\hline CANBERRA & Olen & $\begin{array}{c}\text { Si implanted, Si-drift } \\
\text { readout }\end{array}$ & P. Burger \\
\hline CIS & Erfurt & Si implanted & R. Röder \\
\hline CSEM COLIBRI & Neuchatel & Si implanted & P. Weiss \\
\hline EURISYS & $\begin{array}{l}\text { Strasbourg/ } \\
\text { Lingolsheim }\end{array}$ & $\begin{array}{l}\text { Si implanted, } \\
\text { readout modules }\end{array}$ & M.O.Lampert \\
\hline HAMAMATSU & Hamamatsu & Si implanted & K.Yamamoto \\
\hline MICRON & Southampton & Si implanted & C. Wilburn \\
\hline ORTEC & Oak Ridge & $\begin{array}{l}\text { Si surface barrier, } \mathrm{Si}(\mathrm{Li}) \text {, } \\
\text { implanted, readout modules }\end{array}$ & M. Martini \\
\hline SINTEF & Oslo & Si implanted & B. Avset \\
\hline
\end{tabular}

Only rarely and on special occasions in this journal one may find this kind of description of people behind the methods and instruments in nuclear science. The publications of results should normally speak for themselves, without emphasizing the personal aspects, originality, circumstances and difficulties. However on the formal retirement of Dr Paul Burger from Canberra Semiconductor NV it is appropriate to dedicate an article describing some aspects of his contributions to the developments in silicon detector technology, which have enormously widened the fields of application. There is even more reason for this because most of his continuous efforts have remained outside the litterature. One factor has been the confidential nature of manufacturing details, another is related to conventions in the scientific world. Papers on nuclear particle detectors in general, and silicon diode detectors in 
particular are usually published by scientists from research institutes and universities, and only a small fraction originates from the industrial manufacturers of semiconductor detectors. Even if essential work by an industrial partner contributed to the results, often this is hardly mentioned, and only exceptionally the collaborators from industry co-author the publications. In many such cases the industrial partner is requested to execute tasks as defined precisely in a purchase work order, drawn up by the research scientist. It seems as if not much inventivity is asked from the industrial partner. In practice often this is not true and a dialogue develops on major design aspects, leading to adaptations to the industrial manufacturing environment. For once, the role of the detector manufacturer may be put in the limelight, as a part of these Proceedings of the Workshop on Radiation Imagers.

\section{Conclusion}

The introduction of planar processing and oxide isolation has profoundly changed the use of silicon particle detectors. Originally, single diode devices were primarily applied for rate measurements and energy spectrometry. In the form of segmented matrices $\mathrm{Si}$ devices can now be used as radiation imaging sensors. They can 'look' from a distance at radiation emitting sources such as stars, galaxies or nuclear reactors, or they can be placed in elementary particle experiments where they sense in 3D space the reaction products as these traverse in all directions. The measured coordinates allow the reconstruction of the physics processes that took place. At this time one is operating or building up several large silicon detector systems for particle physics and for the study of particles from outer space before these are transformed by interactions in the atmosphere. Only with industrial manufacturing it has been possible to construct such large areas of silicon detectors.

\section{Acknowledgements}

The author thanks the Organizing Committee of the 9th IWoRID for the invitation to present some historical aspects of the development of silicon radiation imaging detectors. Numerous intellectual exchanges with all industrial manufacturers have contributed in an essential way to the increased use of silicon detectors, and the author is grateful to have been deeply involved in these contacts. It is appropriate to mention that many people around the world dedicate much of their life and continue to contribute to this virtuous spiral of technology innovation and scientific discovery.

\section{References}

[1] W.D. Davis, Silicon crystal counters, J. Appl. Phys. 29 (1958) 231

[2] P.J. van Heerden, The crystal counter, a new instrument in nuclear physics, PhD Thesis, Utrecht 1945, and P.J. van Heerden and J.M.W. Milatz, The crystal counter - parts I and II, Physica 16 (1950)

[3] K.G. McKay, A germanium counter, Phys Rev 76 (1949)

[4] J.W. Mayer, The development of the junction detector, IRE Trans Nucl Sci NS-7 (June 1960) 178

[5] T.C. Madden and W.M. Gibson, Silicon dioxide passivation of p-n junction particle detectors, Rev. Sci. Instrum. 34 (1963) 50 and W.M. Gibson, Oxide edge protection, in [6], p.232

[6] E. Dabbs and F.J. Walter, editors, Nat.Ac. Sciences-NRC Publ. 871, Proc. Informal Conf Asheville, NC, Sept

T.C. Madden and G.L. Miller, editors, Nat. Ac. Sciences, Publ. 1593, Washington, 1969 p.314 Gatlinburg meeting 1968

[8] T. Alväger and N.J. Hansen, Doping of crystals by ion bombardment tp produce solid state detectors, Rev. Sci. Instrum. 33 (1962) 567 
[9] O. Meyer and G. Haushahn, High resolution particle-detectors produced by ion-implantation, Nucl. Instr. Meth. 56 (1967) 177

[10] J.W. Mayer, Use of ion implantation techniques to fabricate semiconductor nuclear particle detectors, Nucl. Instr. Meth. 63 (1968) 141

[11] P. Zandveld, Some properties of ion-implanted p-n junctions in silicon, Solid-St. Electron. 19 (1976) 659 and Paul Zandveld, Method of manufacturing a semiconductor device by implanting ions through bevelled oxide layer in single masking step, US Patent 4,104,085 filed 3 Nov 1976 (priority NL 11 Nov 1975)

[12] J. Kemmer, Fabrication of low noise silicon radiation detectors by the planar process, Nucl. Instr. Meth. 169 (1980) 499

[13] Josef Kemmer, Passivated semiconductor PN junction of high electric strength and process for the production thereof, US Patent 4,442,592 filed 14 Jan 1981 (priority DE 31 jan 1980)

[14] E.H.M. Heijne, L. Hubbeling, B.D. Hyams, P. Jarron, P. Lazeyras, F. Piuz, J.C. Vermeulen and A. Wylie, A silicon surface barrier microstrip detector designed for high energy physics, Nucl. Instr. Meth. 178 (1980) 331

[15] J. Kemmer, P. Burger, R. Henck and E. Heijne, Performance and applications of passivated ion-implanted silicon detectors, IEEE Trans. Nucl. Sci. NS-29 (1982) 733

[16] D.P. Oosthoek and E. Kok, Semiconductor device for detecting and/or measuring radiation, US Patent 3529 161, 1970 (priority NL March 1, 1966)

[17] W.K.Hofker, D.P. Oosthoek, A.M.E. Hoeberechts, R. van Dantzig, K. Mulder, J.E.J. Oberski, L.A.Ch. Koerts, J.H. Dieperink, E. Kok and R.F. Rumphorst, The checker board counter: a semiconductor dE/dx detector with position indication, IEEE Trans Nucl Sci NS-13 (June 1966)

[18] S.R. Amendolia, G. Batignani, F. Bedeschi, E. Bertolucci, L. Bosisio, C. Bradaschia, M. Budinich, F. Fidecaro, L.Foa, E. Focardi, A. Giazotto, M.A. Giorgi, M. Givoletti, P.S. Marrocchesi, A. Menzione, D. Passuello, M. Quaglia, L. Ristori, L. Rolandi, P. Salvadori, A. Scribano, R. Stanga, A. Stefanini and M.L. Vincelli, A multi - electrode silicon detector for high energy physics experiments, Nucl. Instr. Meth. 176 (1980) 457

[19] L.A.Ch. Koerts, K. Mulder, J.E.J. Oberski and R. van Dantzig, The 'BOL' nuclear research project, Nucl. Instr. Meth. 92 (1971) 157 and following articles in that issue

[20] A. Stefanini, editor, Miniaturization of High-Energy Physics Detectors, Proceedings first 'Pisa Conference in Tirennia, Sept 1980' Plenum Press, New York (1983)

[21] Tom Ferbel (Rochester U.), editor, Silicon Detectors For High-Energy Physics. Proceedings, Workshop, Batavia, USA, October 15-16, 1981, Batavia, USA: Fermilab ( 1982)

[22] G. Bellini and S.C.C. Ting, editors, in "The search for charm, beauty and truth at high energies", Europhysics Study Conf Erice, Nov 1981 Plenum Publ.Corp, New York (1984)

[23] B. Hyams, U. Koetz, E. Belau, R. Klanner, G. Lutz, E. Neugebauer, A. Wylie and J. Kemmer, A silicon counter telescope to study short-lived particles in high-energy hadronic interactions, Nucl. Instr. Meth. 205 (1983) 99

[24] E. Belau, J. Kemmer, R. Klanner, U. Kötz, G. Lutz, W. Männer, E. Neugebauer, H.J. Seebrunner and A. Wylie, Silicon detectors with $5 \mu \mathrm{m}$ spatial resolution for high energy particles, Nucl. Instr. Meth. 217 (1983) 224

[25] E.H.M. Heijne, R. Klanner, G. Lutz, H.J. Seebrunner and A. Wylie, ed., New developments in silicon detectors, Proc.3rd European Symp. Semiconductor Detectors,14-16 Nov 1983, Munich, Nucl. Instr. Meth. 226 (1984)(1)

[26] J. Kemmer, P. Burger, R. Henck and E.H.M. Heijne, Performance and application in high energy physics of passivated ion-implanted silicon detectors, p.269 in "The search for charm, beauty and truth at high energies", Ed. G. Bellini and S.C.C. Ting, Plenum Publ.Corp, New York (1984)

[27] S. Benso, M. Dameri, G. Diambrini-Palazzi, P.F. Manfredi, D. Marioli, C. Meroni, L. Rossi, M. Sannino, S. Tentindo and G. Vegni, Silicon telescopes as charm decay detectors, Nucl. Instr. Meth. 201 (1982) 329

[28] A development project for Si surface barrier segmented detectors had been discussed with Ortec Inc. in December 1979, T. Ludlam, private communication

[29] A. Kanofsky, Solid state micro-detectors for high energy experiments, Nucl. Instr. Meth. 140 (1977) 429

[30] H.W. Kraner, R. Beuttenmuller, T. Ludlam, A.L. Hanson, K.W. Jone, V. Radeka and E.H.M. Heijne, Charge collection in silicon strip detectors, IEEE Trans. Nucl. Sci. NS-30(1983)405 (Nucl. Sci. Symp. 1982)

[31] M. Bourquin, On behalf of the AMS Tracker Collaboration, The AMS tracking detector for cosmic-ray physics in space, Nucl. Instr. Meth. A541 (2005) 110 STD5 Hiroshima

[32] Alessandro Brez, Construction strategy of the GLAST tracker, Nucl. Instr. Meth. A549 (2005) 178 Vertex 2003

[33] E. Gatti and P. Rehak, Semiconductor drift chamber - an application of a novel charge transport scheme, Nucl. Instr. Meth. 225 (1984) 608

[34] H. Heyns and J.G. van Santen, The resistive gate CTD area-image sensor, IEEE Trans. Electron Dev. ED-25 (1978) 196

[35] Robert N. Noyce, Semiconductor Device-and-Lead Structure, U. S. Patent 2,981,877, filed 6 July 1959, issued 25 April 1961

[36] Jean A. Hoerni, Method of Manufacturing Semiconductor Devices,' U. S. Patent 3,025,589, filed 1 May 1959, issued 20 March 1962

[37] Jack St. Clair Kilby, Miniaturized Electronic Circuits,’U. S. Patent 3,138,743, filed 6 February 1959, issued 23 June 1964

[38] A. Pullia and G. Bertuccio, Resolution limits of silicon detectors and electronics for soft X-ray spectroscopy at non cryogenic temperatures, Nucl. Instr. Meth. A380 (1996) 1 RTSD96

[39] E. Steinbauer, P. Bauer, M. Geretschläger, G. Bortels, J.P. Biersack and P. Burger, Energy resolution of silicon detectors: approaching the physical limit, Nucl. Instr. Meth. B85 (1994) 642

[40] F. Conti and M. Conti, Surface breakdown in silicon planar diodes equipped with field plate, Solid-St. Electron. 15 (1972) 93 
[41] B.J. Baliga, Power semiconductor devices, Boston 1996, PWS Publishing, ISBN 0-534-94098-6

[42] C.J. Kenney, J.D. Segal, E. Westbrook, Sherwood Parker, J. Hasi, C. Da Via, S. Watts and J. Morse, Activeedge planar radiation sensors, Nucl. Instr. Meth. A565 (2006) 272 Pixel2005

[43] Christopher Kenney, Sherwood Parker, Julie Segal, and Chris Storment, IEEE Trans Nucl Sc 46 (1999) 1224, Silicon Detectors with 3-D Electrode Arrays: Fabrication and Initial Test Results

[44] G. Keil and E. Lindner, Low-noise oxide passivated p+n silicon detectors, Nucl. Instr. Meth. 101 (1972) 43 (2nd Munich Symp) and idem, Low-noise silicon planar detectors for room temperature application, Nucl. Instr. Meth. 104 (1972) 209

[45] G. Bertuccio, A. Pullia and G. De Geronimo, Criteria of choice of the front-end transistor for low-noise preamplification of detector signals at sub-microsecond shaping times for X-and $\gamma$-ray spectroscopy, Nucl. Instr. Meth. A380 (1996) 301 RTSD96

[46] K.J. Rawlings, J.W. Leake and S.C. Jain, The application of high-low junctions to silicon radiation detectors, Nucl. Instr. Meth. A245 (1986) 512

[47] E. Laegsgaard, Position-sensitive semiconductor detectors, Nucl. Instr. Meth. 162 (1979) 93 